\title{
BMJ Open Psychosocial consequences of false positives in the Danish Lung Cancer CT Screening Trial: a nested matched cohort study
}

\author{
Jakob Fraes Rasmussen, ${ }^{1}$ Volkert Siersma, ${ }^{1}$ Jessica Malmqvist (D) , ${ }^{1,2}$ \\ John Brodersen ${ }^{1,2}$
}

To cite: Rasmussen JF, Siersma V, Malmqvist J, et al. Psychosocial consequences of false positives in the Danish Lung Cancer CT Screening Trial: a nested matched cohort study. BMJ Open 2020;10:e034682. doi:10.1136/ bmjopen-2019-034682

- Prepublication history and additional material for this paper are available online. To view these files, please visit the journal online (http://dx.doi. org/10.1136/bmjopen-2019034682).

Received 02 0ctober 2019 Revised 06 April 2020 Accepted 04 May 2020
Check for updates

(c) Author(s) (or their employer(s)) 2020. Re-use permitted under CC BY-NC. No commercial re-use. See rights and permissions. Published by BMJ.

${ }^{1}$ The Research Unit for General Practice and Section of General Practice, Department of Public Health, University of Copenhagen, Copenhagen, Denmark

${ }^{2}$ Primary Health Care Research Unit, Region Zealand, Region Zealand, Denmark

Correspondence to Dr Jessica Malmqvist; jessica.malmqvist@sund.ku.dk

\section{ABSTRACT}

Objectives Lung cancer CT screening can reduce lung cancer mortality, but high false-positive rates may cause adverse psychosocial consequences. The aim was to analyse the psychosocial consequences of false-positive lung cancer CT screening using the lung cancer screeningspecific questionnaire, Consequences of Screening in Lung Cancer (COS-LC).

Design and setting This study was a matched cohort study, nested in the randomised Danish Lung Cancer Screening Trial (DLCST).

Participants Our study included all 130 participants in the DLCST with positive CT results in screening rounds $2-5$, who had completed the COS-LC questionnaire. Participants were split into a true-positive and a false-positive group and were then matched $1: 2$ with a control group $(n=248)$ on sex, age ( \pm 3 years) and the time of screening for the positive CT groups or clinic visit for the control group. The true positives and false positives were also matched $1: 2$ with participants with negative CT screening results $(\mathrm{n}=252)$.

Primary outcomes Primary outcomes were psychosocial consequences measured at five time points.

Results False positives experienced significantly more negative psychosocial consequences in seven outcomes at 1 week and in three outcomes at 1 month compared with the control group and the true-negative group (mean $\Delta$ score $>0$ and $p<0.001$ ). True positives experienced significantly more negative psychosocial consequences in one outcome at 1 week (mean $\Delta$ score $2.86(95 \% \mathrm{Cl} 1.01$ to 4.70$), p=0.0024)$ and in five outcomes at 1 month (mean $\Delta$ score $>0$ and $p<0.004)$ compared with the true-negative group and the control group. No long-term psychosocial consequences were identified either in false positives or true positives. Conclusions Receiving a false-positive result in lung cancer screening was associated with negative short-term psychosocial consequences. These findings contribute to the evidence on harms of screening and should be taken into account when considering implementation of lung cancer screening programmes.

Trial registration number NCT00496977.

\section{INTRODUCTION}

Lung cancer has the highest mortality worldwide. ${ }^{1}$ Several randomised controlled
Strengths and limitations of this study

- This study used a lung cancer screening-specific questionnaire with high content validity and adequate psychometric properties to measure the psychosocial consequences of the screening results.

- In addition to the false-positive group, the truepositive group and the true-negative group were assessed, serving as benchmarks against which to compare the psychosocial consequences in the false positives.

- A limitation is that the control group, who were not invited to screening, reported more negative psychosocial consequences than the screening group.

- Another limitation is that the study participants had a more robust psychosocial profile compared with a matched background population.

screening trials using low-dose CT scans have investigated the effect of CT screening on lung cancer-specific mortality. ${ }^{2}$ The largest trial, the National Lung Screening Trial (NLST), found a relative lung cancer-specific mortality reduction of $16 \%$ after 5-year follow-up, and lung cancer CT screening is now recommended in the USA. ${ }^{3-5}$ However, according to a Cochrane systematic review, more data are needed on false-positive results and overdiagnosis before recommendations can be made for large-scale CT-screening programmes. $^{6}$ The Danish Lung Cancer Screening Trial (DLCST) could not show a reduction in lung cancer-specific or total mortality after a 5 -year follow-up. ${ }^{7}$ The European trials are expected to publish the pooled follow-up analyses of both the mortality data and the consequences of overdiagnosis and false-positive results. ${ }^{8}$ This will provide the additional evidence of benefits and harms of lung cancer CT screening requested in the Cochrane systematic review. ${ }^{6}$ 
In cancer screening programmes, positive screening results lead to either false-positive results or true-positive results after further diagnostic workup. ${ }^{9} \mathrm{~A}$ false-positive screening result can cause both physical and psychosocial harms ${ }^{10-13}$ as well as being costly for the healthcare system. ${ }^{14-16}$ The average false-positive rate per screening round varies substantially in lung cancer screening trials, for example, $23 \%$ in the NLST and $3 \%$ in the DLCST (online supplementary appendix 1). ${ }^{317}$ Qualitative and quantitative studies have shown that false-positive lung cancer screening results can be associated with negative psychosocial consequences both during workup and after the final diagnosis. ${ }^{13} 1819$ By their nature, qualitative studies cannot measure the degree or the extent of psychosocial consequences ${ }^{18}$ and all the published quantitative studies used generic questionnaires which lack content validity and have unknown psychometric properties. ${ }^{13}$ 19-21 Measurement of the psychosocial consequences of screening using questionnaires with high content validity and adequate psychometric properties is important. ${ }^{22}$

The aim of this study, therefore, was to measure the short-term and long-term psychosocial consequences of false-positive lung cancer CT screening results using the questionnaire Consequences of Screening in Lung Cancer (COS-LC) and to compare these scores with the COS-LC scores from three other groups of participants in the DLCST: 1) the true-negative group, 2) the truepositive group and 3) a control group that did not participate in screening.

\section{METHODS}

\section{Study design and participants}

The overall design of the DLCST has been reported in detail elsewhere. ${ }^{1723}$ In summary, the DLCST was a singlecentre, randomised controlled trial and participants were randomly allocated to a CT group and a control group

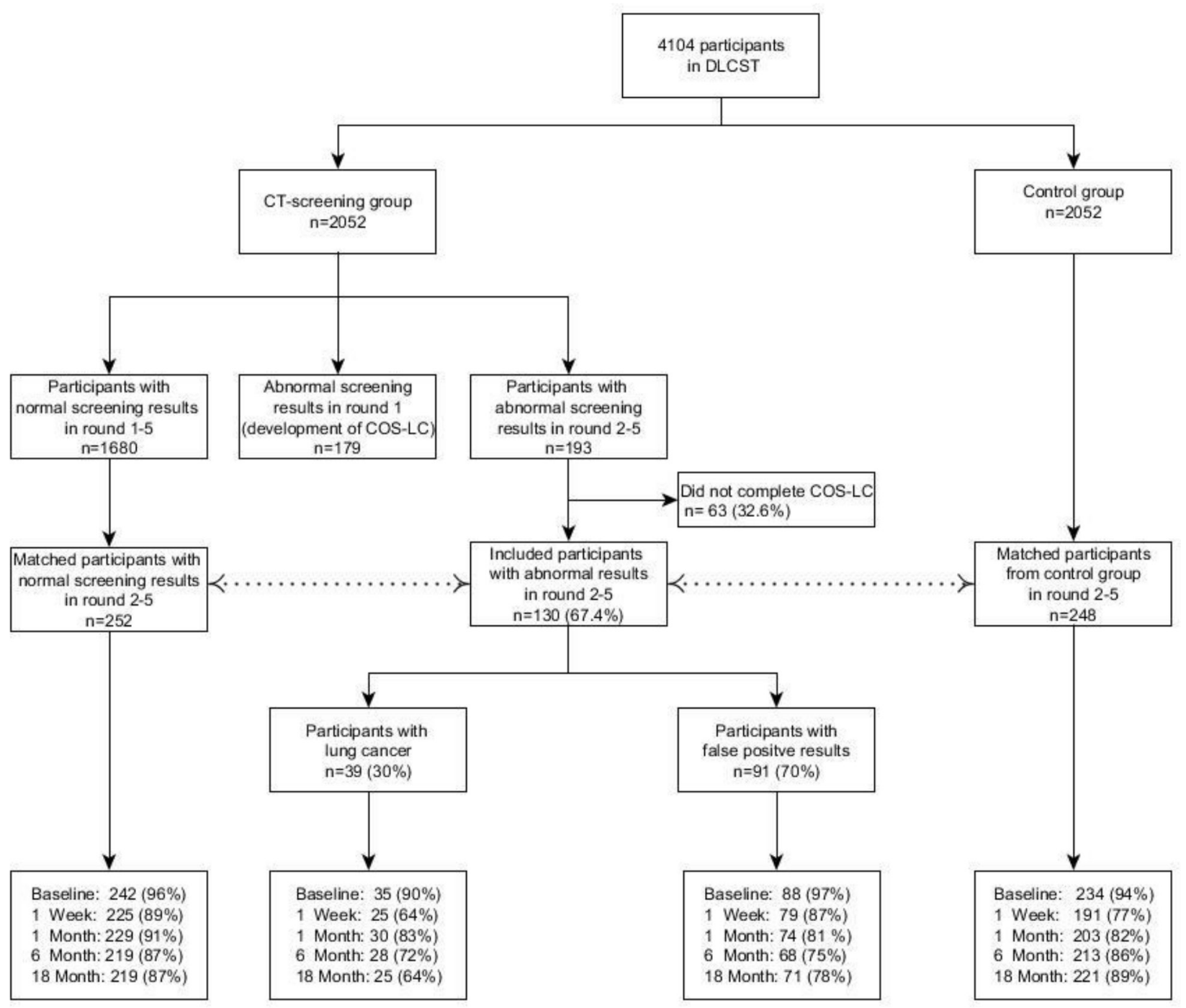

Figure 1 Distribution of screening results and final diagnoses in the DLCST, and response rates of the matched groups at five time points: baseline, 1 week, 1, 6 and 18 months. COS-LC, Consequences of Screening in Lung Cancer; DLCST, Danish Lung Cancer Screening Trial. 
(figure 1). Eligible participants were current and former smokers with a smoking history of minimum 20 cigarettes/day for 20 years, and were aged 50-70 years. ${ }^{1723}$ In five rounds between 2004 and 2010, both groups were offered annual spirometry and smoking counselling and were asked to complete the COS-LC questionnaire. Participants in the CT group were also offered annual lung CT scans.

This study was a matched cohort study nested in the DLCST. Participants from the CT group with positive CT screening results during rounds $2-5$ were matched 1:2 with participants with negative CT screening results, and 1:2 with participants from the control group. Participants were matched on sex, age ( \pm 3 years) and the time of screening, within 7 days for the CT group, or clinic visit for the control group. The group with positive CT screening results was further divided into a true-positive group and a false-positive group after receiving the final diagnosis. Participants completed the COS-LC at five time points (figure 1):

- Baseline: COS-LC was completed shortly before the annual CT screening (CT group) or clinic visit (control group).

- One week after receiving the CT screening result (CT group) and 1 week after the annual clinic visit (control group).

- 1,6 and 18 months after receiving the final diagnosis of the screening result (CT group) and at these time points after the annual clinic visit (control group).

At the latter four time points, participants were sent the COS-LC by post and asked to return it in an enclosed stamped addressed envelope. A reminder was sent to participants who did not return the COS-LC within 2 weeks.

Information about region of residence, smoking status, smoking history, social group, employment status, school education and whether participants lived alone was obtained from baseline and annual questionnaires. The Charlson Comorbidity Index was calculated from hospital admissions 3 years before baseline.

\section{Questionnaire}

The COS-LC is a condition-specific questionnaire with high content validity and adequate psychometric properties and it was developed and validated to measure the psychosocial consequences of participation in lung cancer CT screening. ${ }^{18}$ To ensure high content validity, 20 participants from the first screening round in the DLCST were interviewed in five group interviews. ${ }^{18}$ Subsequently, during screening rounds 2-4 in the years 2006-2007, questionnaire data from participants were used to validate the COS-LC using Item Response Theory Rasch models. ${ }^{18}$ As these data were only a part of the present data, the psychometric properties of the 15 COS-LC scales were re-tested for homogeneity and differential item functioning (DIF) relative to participant group, sex, age, social status and smoking status by using likelihood ratio tests on appropriately conditioned Rasch models at the 1 month follow-up time point. ${ }^{24}$ Reliability of the scales was examined using Cronbach's alpha.

The COS-LC has two parts where part I encompasses 24 COS items (4 COS scales) and 25 lung cancer screeningspecific items (5 lung cancer screening-specific scales) (online supplementary appendix 2). Part I can be used before, during and after screening and the DLCST participants in both the CT group and the control group completed part I. ${ }^{18}$ The higher the scale score, the more negative the psychosocial consequences. ${ }^{18}$

Part II measures the long-term psychosocial consequences after lung cancer CT screening and can therefore only be completed by the screening participants (CT group) after they have received their final diagnosis. ${ }^{18}$

Part II encompasses 24 items (6 scales) and was designed and validated to measure changes, both positive and negative; high scores denote more change (online supplementary appendix 2).

\section{Statistical analysis}

The differences in the characteristics of the four groups of participants (true-negative, true-positive, false-positive and control) were tested with Pearson's $\chi^{2}$ tests for categorical variables and Kruskal-Wallis non-parametric tests for continuous variables.

For each of the 15 COS-LC scales, the mean score for each of the four participant groups at the five time points was analysed with linear regression models, both unadjusted and adjusted for the participant characteristics: round, sex, (a quadratic function of) age, region, (a quadratic function of) pack years, smoking status, social group, living alone, employment status, school education and Charlson Comorbidity Index. Generalised estimating equations were used to account for repeated measurement. To adjust for differential dropout, the non-missing scales at each time point were weighted by the inverse of the probability of this scale being observed at that time. ${ }^{25}$ These probabilities were estimated from the data in logistic regression models for the scale being missing, which included the participant characteristics, the participant groups and the corresponding scale outcomes from previous time points.

The statistical level of significance was set using the method of Benjamini-Hochberg to adjust for multiple testing. ${ }^{26}$ Statistical Analysis Software V.9.3 was used to analyse the data.

\section{Participant and public involvement}

DLCST participants were involved in the development of the questionnaire COS-LC. Neither participants nor the Danish general public were involved in the design and recruitment of the study.

\section{RESULTS}

\section{Participation}

Distribution of final diagnostic results and participation rates are presented in figure 1. In rounds 2-5, 193 
participants received a positive screening result; of those, $130(67 \%)$ completed the COS-LC and were included in this study. The reasons for non-response were: 1) never receiving the COS-LC because the participant contact details were not available to the researchers $(n=39,20 \%)$, 2) declining to complete the COS-LC $(n=6,3 \%)$; and 3$)$ other reasons $(n=18,9 \%)$.

Of the 130 respondents included in the study, 24 (19\%) had received one false-positive result in the previous rounds and one $(0.8 \%)$ had previously received two falsepositive results. The COS-LC was sent to 252 participants with true-negative results and 248 control participants. Response rates for the four groups during the five time points were $64 \%-97 \%$ (figure 1).

There was a significant difference between the four groups regarding age and smoking history: the participants in the true-positive group were older and had a longer smoking history (table 1). A significant difference was also observed in the region of residence, where false positives, to a greater extent, lived outside the capital region compared with the other groups. No significant differences were found in the remaining participant characteristics.

The 15 COS-LC scales exhibited overall adequate fit to the partial credit Rasch model for polytomous items. No DIF was revealed and Cronbach's alpha was 0.693-0.962 (table 2).

\section{COS-LC part I}

Figure 2 presents the mean score of the nine outcomes for COS-LC part I for the four groups at the five time points. For part I in general, participants with a positive CT screening result reported more negative psychosocial consequences at the short-term follow-up points of 1 week and 1 month (figure 2). The false-positive group experienced significantly more negative psychosocial consequences at 1 week in seven outcomes (anxiety, behaviour, dejection, self-blame, focus on airway symptoms, introvert and harm of smoking) and at 1 month in three outcomes (self-blame, focus on airway symptoms and harm of smoking) (mean $\Delta$ score $>0$ and $\mathrm{p}<0.001$ ) compared with both the control group and the truenegative group (figure 2, online supplementary appendix 3). At 6 and 18 months, there was a trend towards more negative psychosocial consequences in three outcomes, but no statistically significant differences were found. The true-positive group showed the same general pattern and experienced significantly more negative psychosocial consequences only in the outcome dejection at 1 week (mean $\Delta$ score 2.86 (95\% CI 1.01 to 4.70 ), $\mathrm{p}=0.0024$ ) and in the three outcomes behaviour, dejection and focus on airway symptoms at 1 month (mean $\Delta$ score $>0$ and $\mathrm{p}<0.004)$ compared with the true-negative group and the control group (figure 2, online supplementary appendix $3)$. At baseline, the true-positive group showed a significantly more positive psychosocial profile in the outcomes anxiety and self-blame.

\section{COS-LC part II}

Figure 3 presents the mean scores of the six outcomes for COS-LC part II for the three groups at the three follow-up points after receiving the final screening result. The false-positive group showed a trend towards more psychosocial consequences in two outcomes at 1 month compared with the true-negative group, but no significant differences were seen. The true-positive group showed significant differences in the outcome social network at 1 month and 6 months and in the outcome empathy at 1 month (figure 3, online supplementary appendix 3). Trends towards more psychosocial consequences were seen in five outcomes at 1 month compared with the truenegative group. This difference diminished at 6 and 18 months. The true-negative group showed no variation in psychosocial consequences through the three long-term follow-up points.

\section{DISCUSSION}

False-positive lung cancer CT screening results were associated with negative short-term psychosocial consequences compared with the control group and the true-negative group. There were no identified long-term consequences of false-positive results. Contrary to expectation, neither were there any long-term consequences experienced by the true-positive group.

The tendency towards more negative long-term psychosocial consequences in the false-positive group was limited to three lung cancer-specific scales in part I of COS-LC. The same pattern was seen for the true-positive group. Additionally, this group reported more psychosocial consequences in the scales Social Network and Empathy in part II of COS-LC (figure 3). Smoking causes approximately $90 \%$ of all lung cancers and on a societal level smokers are often blamed for their lung cancer, which can lead to feelings of self-blame and guilt. ${ }^{27}$ This could explain the tendency towards long-term negative psychosocial consequences in the lung cancer-specific scales: self-blame, focus on airway symptoms and harm of smoking in part I. In contrast, no negative long-term consequences were seen in the remaining six scales in part I. There might be several explanations for our findings: (1) the true-positive group had a more positive psychosocial profile at baseline than the other groups. Hence, no long-term differences compared with the control group were seen, when the short-term negative psychosocial consequences diminished with time towards the more positive set point; (2) selection bias was identified among DLCST participants, who were better educated and with a more positive psychosocial profile compared with a matched background population. ${ }^{28}$ Thus, DLCST participants were probably more psychosocially robust than average and therefore false-positive or true-positive findings might have had fewer negative consequences than could be expected for the general population; (3) those diagnosed with lung cancer via screening and who remained alive and asymptomatic after 18 months were 
Table 1 Characteristics of participants

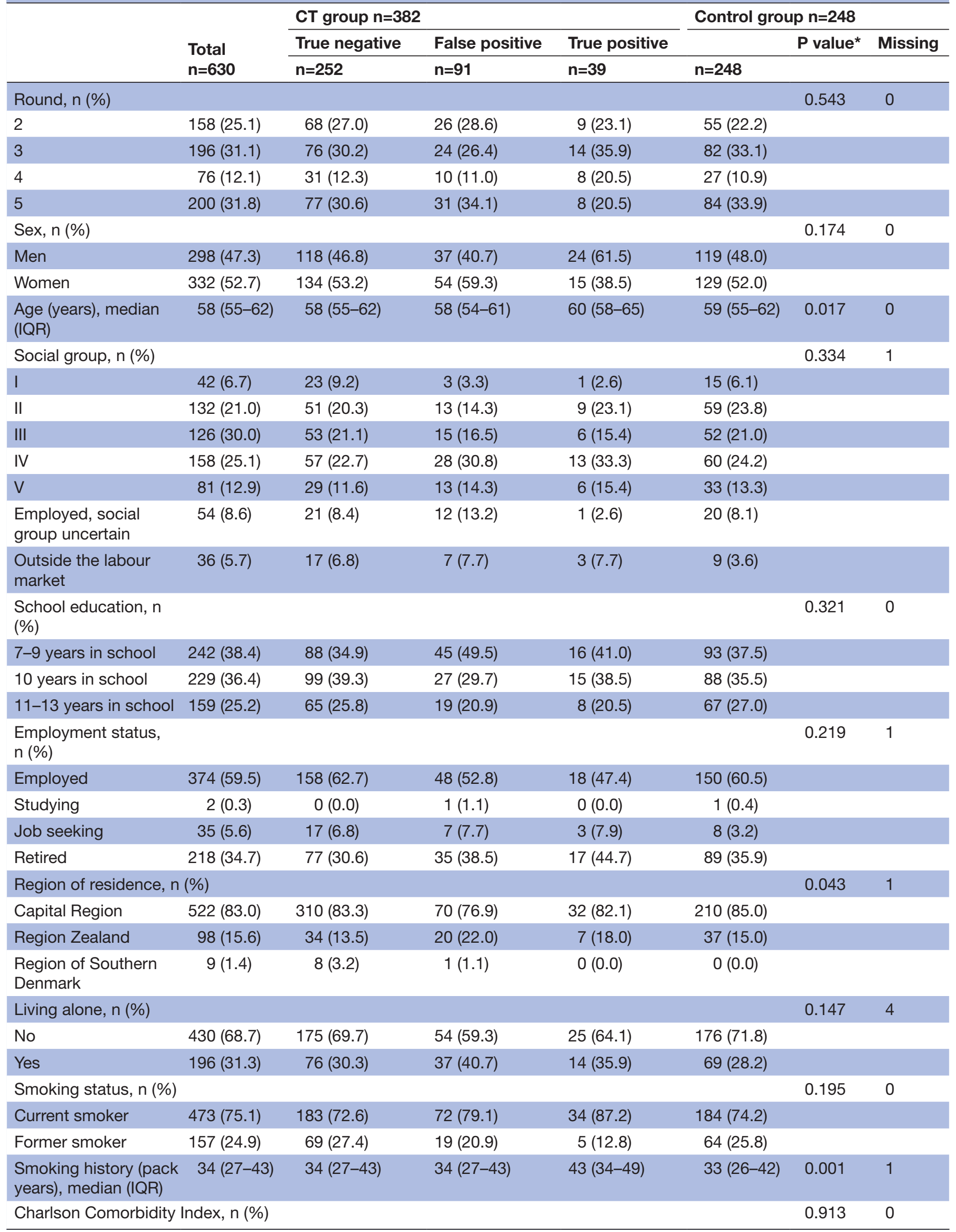




\begin{tabular}{|c|c|c|c|c|c|c|c|}
\hline & \multirow{3}{*}{$\begin{array}{l}\text { Total } \\
n=630\end{array}$} & \multicolumn{3}{|c|}{ CT group $n=382$} & \multicolumn{3}{|c|}{ Control group $n=248$} \\
\hline & & True negative & False positive & True positive & & P value* & Missing \\
\hline & & $\mathrm{n}=252$ & $\mathrm{n}=91$ & $\mathrm{n}=39$ & $\mathrm{n}=\mathbf{2 4 8}$ & & \\
\hline 1 & $25(4.0)$ & $10(4.0)$ & $5(5.5)$ & $2(5.1)$ & $8(3.2)$ & & \\
\hline$\geq 2$ & $15(2.4)$ & $7(2.8)$ & $3(3.3)$ & $1(2.6)$ & $4(1.6)$ & & \\
\hline
\end{tabular}

${ }^{*} \mathrm{P}$ value of a Pearson's $\chi^{2}$ test (categorical variables) or a Kruskal-Wallis test (continuous variables); $p$ values are estimates of the exact $p$ values based on 10000 Monte Carlo simulations under the null-hypothesis.

convinced, they had been cured of a lethal disease. This reassurance is likely since lung cancer symptom lead time is longer than 18 months and a minimum of $20 \%$ of screening detected lung cancers are overdiagnosed. ${ }^{29}$ If those diagnosed with lung cancer via screening do not experience any substantial long-term negative psychosocial consequences, it is not expected that the experiences of other screening groups will differ; (4) another explanation for the long-term results could be the fact that the control group experienced more negative psychosocial consequences than the CT group through screening rounds $2-5$ in DLCST. ${ }^{30}$ The level of psychosocial consequences in the control group was therefore more negative (higher COS-LC scores), which decreases the difference

Table 2 Conditional likelihood ratio (CLR) fit statistics and Cronbach's alpha for the 15 domains of the Consequences of Screening in Lung Cancer questionnaire

\begin{tabular}{|c|c|c|c|c|}
\hline Scales (no. of items) & CLR & df & P value* & $\begin{array}{l}\text { Cronbach's } \\
\alpha\end{array}$ \\
\hline Anxiety (7) & 23.0 & 20 & 0.286 & 0.903 \\
\hline Behaviour (7) & 19.0 & 20 & 0.520 & 0.893 \\
\hline Dejection (6) & 14.9 & 17 & 0.603 & 0.916 \\
\hline $\begin{array}{l}\text { Negative impact on } \\
\text { sleep (4) }\end{array}$ & 22.3 & 11 & 0.022 & 0.874 \\
\hline Self-blame (5) & 20.2 & 14 & 0.124 & 0.962 \\
\hline $\begin{array}{l}\text { Focus on airway } \\
\text { symptoms (2) }\end{array}$ & 1.0 & 5 & 0.966 & 0.802 \\
\hline Stigmatisation (4) & 24.6 & 11 & 0.010 & 0.916 \\
\hline Introvert (4) & 11.2 & 11 & 0.425 & 0.851 \\
\hline Harm of smoking (2) & 9.8 & 5 & 0.082 & 0.857 \\
\hline Existential values (6) & 9.3 & 11 & 0.591 & 0.851 \\
\hline Calm/Relaxed (2) & 0.6 & 3 & 0.887 & 0.693 \\
\hline Social network (3) & 5.5 & 5 & 0.362 & 0.754 \\
\hline Impulsivity (6) & 4.5 & 11 & 0.954 & 0.854 \\
\hline Empathy (3) & 5.9 & 5 & 0.314 & 0.699 \\
\hline $\begin{array}{l}\text { Regretful about still } \\
\text { smoking (4) }\end{array}$ & 1.0 & 7 & 0.795 & 0.863 \\
\hline
\end{tabular}

${ }^{*}$ After adjustment for multiple testing by using the methods of Benjamini-Hochberg, the level of statistical significance was assessed at 0.0033 . between the control group and the positive CT screening groups; (5) during the development of the COS-LC, the qualitative interviews were conducted $0-5$ months after screening; therefore, part II of the COS-LC might not capture all relevant long-term psychosocial consequences for those with false-positive findings; (6) approximately $20 \%$ of the participants who received a positive screening result had previously received a false-positive result. Participants might therefore become accustomed to receiving a (false-) positive screening result, which could decrease the level of negative psychosocial consequences. In contrast, the COS-LC was developed in the first round and a first-round effect, which most likely would have had a more negative psychosocial impact on the participants, was not seen; (7) contamination of the control group could have biassed our results; nevertheless, contamination of the DLCST was found to be minor ${ }^{31}$; (8) participants with false-positive results could have received a negative screening result between the 6-month and the 18-month assessments, which could be perceived as reassurance, consequently lowering the negative psychosocial consequences.

This is the first study to present both short-term and long-term psychosocial consequences of false-positive results using a lung cancer-specific questionnaire with high content validity and adequate psychometric properties developed in a randomised controlled lung cancer CT screening trial. Therefore, the COS-LC most likely presents stronger results compared with generic questionnaires. The true-positive group was included in this study and when both the true-positive and the truenegative groups are included, the extent of the psychosocial harm in the false-positive group can be compared with the extent of harm in those who should be worst off (true positives) and those who are reassured (true negatives). No significant differences were shown, however, in the long-term psychosocial consequences for either the false-positive group or the true-positive group compared with the control group.

Other quantitative studies have investigated the healthrelated quality of life (HRQoL) in CT screening using generic questionnaires that have not been validated for lung cancer. ${ }^{13}{ }^{19-21}$ Although one lung cancer-specific questionnaire was used, no information about validation was reported. ${ }^{19}$ These studies found that CT screening 


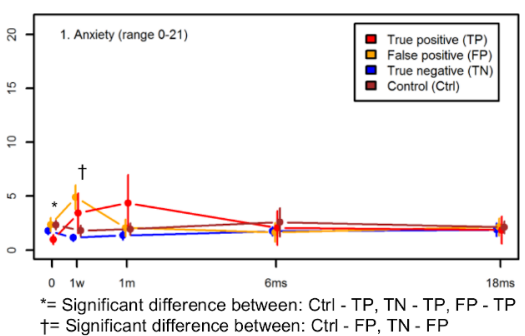

$\dagger=$ Significant difference between: Ctrl - FP, TN - FP
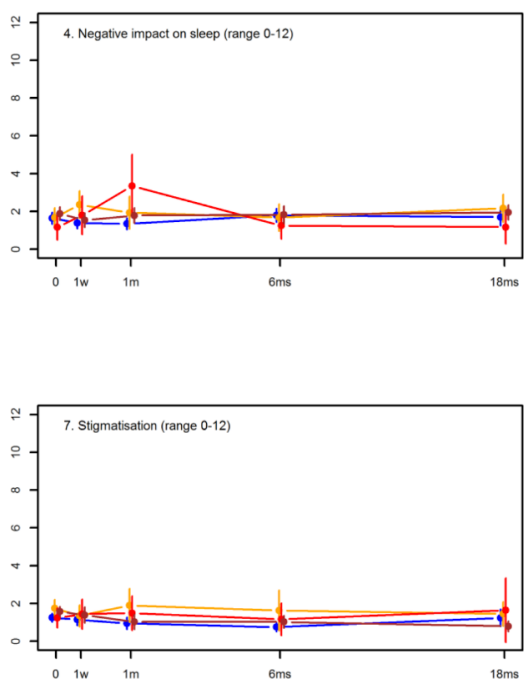

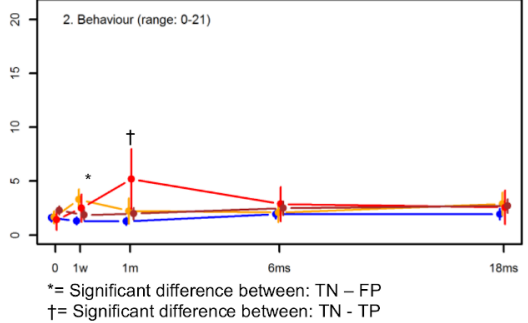

$t=$ Significant difference between: TN - TP
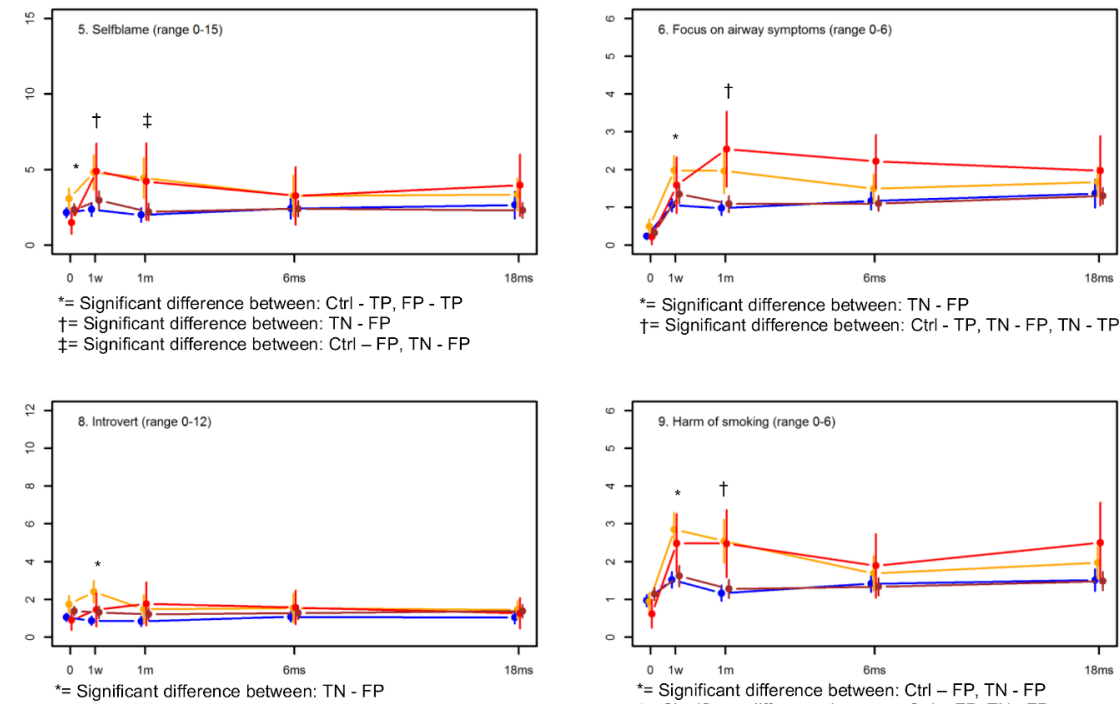

Figure 2 The mean score of the nine psychosocial outcomes of Consequences of Screening in Lung Cancer (COS-LC) part I for the diagnostic groups and the control group in the Danish Lung Cancer Screening Trial at five time points: baseline, 1 week, 1, 6 and 18 months. The means of COS-LC part I scales are compared between all groups at each time point and significant differences between the groups are described below each scale (see online supplementary appendix 1 for details of the adjusted analyses). After adjustment for multiple testing by the method of Benjamini-Hochberg, the level of statistical significance was assessed at 0.0043; $0=$ baseline; $1 \mathrm{w}=1$ week after screening; $1 \mathrm{~m}, 6 \mathrm{~ms}$ and $18 \mathrm{~ms}=1,6$ and 18 months after final diagnostic result; the higher the score the more negative psychosocial reaction.

had only short-term and no long-term negative effects on HRQoL for participants with false-positive results. Our study, using a more accurate and validated survey instrument, has confirmed this. However, the absence of long-term psychosocial consequences in the true-positive group as well suggests that certain long-term consequences may have been overlooked or that the development of a certain resilience or relief (at feeling cured) may play a long-term role. ${ }^{32}$

A study investigating the risk of receiving a prescription on antidepressants or anxiolytics in the CT group (mixed negative and positive results) compared with the control group in DLCST found no differences between these groups. ${ }^{33}$ These outcomes measure extremes of psychosocial consequences, which is a plausible explanation for the negative results. However, another study, which investigated healthcare use and costs in DLCST participants showed higher use of the healthcare system among false positives and true positives compared with the control group in the time period between two screening rounds. ${ }^{15}$ This may be associated with an increased attention drawn to the risk of not being healthy subsequent to receiving a false-positive result. A meta-analysis of the psychosocial consequences of false-positive mammograms including both generic and condition-specific outcome measures showed both short-term and long-term (up to 3 years) negative psychosocial consequences compared with truenegative mammograms. ${ }^{12} 34$ This study recommends the use and further development of condition-specific measures instead of generic measures in mammography screening. Condition-specific measures should also be improved and used in lung cancer CT-screening to obtain the most valid results for psychosocial outcomes.

In interpreting the effect size of the results, we used the mean increase of 2.16 in self-blame in the falsepositive group at the 1 month time point compared with the control group (online supplementary appendix 3). This increase corresponds to two shifts in the response category of one item for all participants with false-positive results, for example, from 'not at all' to 'quite a bit', while all the participants in the control group had no shift in response category. The false-positive rates differ substantially in the NLST (23\%) and the DLCST (3\%), which has been discussed in detail previously. ${ }^{15}$ The negative consequences may seem small or transient in relative terms; however, in absolute terms they might be large. 

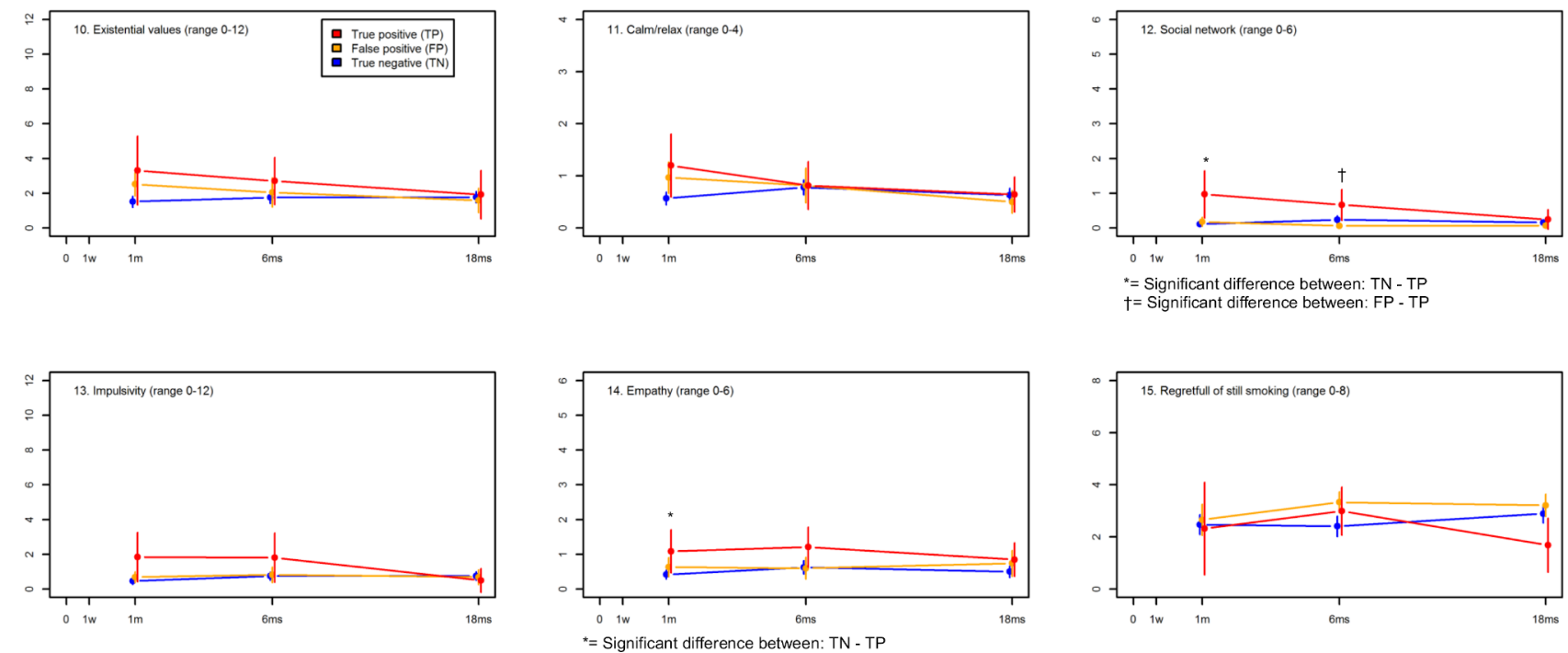

Figure 3 The mean score of the six psychosocial outcomes of Consequences of Screening in Lung Cancer (COS-LC) part II for the diagnostic groups in the Danish Lung Cancer Screening Trial at three time points: 1, 6 and 18 months. The means of COS-LS Part II scales are compared between the three screened groups at each time point after the final diagnostic result and significant differences between the groups are described below each scale (see online supplementary appendix 1 for details of the adjusted analyses). After adjustment for multiple testing by the method of Benjamini-Hochberg, the level of statistical significance was assessed at 0.0043; 0=baseline; $1 \mathrm{w}=1$ week after screening; $1 \mathrm{~m}, 6 \mathrm{~ms}$ and $18 \mathrm{~ms}=1,6$ and $18 \mathrm{months}$ after final diagnostic result; the higher the score the more psychosocial reaction.

There are two reasons for this: first, a mass screening programme targeting a large population, a small change in the frequency with which they appear, may be a large increase in the absolute number of presumably healthy people affected by these consequences; second, the positive predictive value (PPV) of an abnormal low-dose CT screening result might be much lower in an ongoing screening programme compared with the PPV obtained in a research setting, for example, in the Veterans Health Administration in the USA there was nearly a falsepositive rate of $60 \%$ in their first screening round. ${ }^{35}$ This will also increase the costs even more. We have in previous research shown that low-dose CT screening for lung cancer will in a Danish context with a public funded healthcare system increase total health costs by $60 \%{ }^{16}$ and specifically for those with false-positive results the cost will increase by $66 \%{ }^{15}$ The knowledge of psychosocial consequences from false-positive results contributes to the evidence for the benefits and harms of lung cancer CT screening and should be included in the overall assessment of the European trials.

\section{CONCLUSION}

In the DLCST, false-positive results were associated with more negative short-term psychosocial consequences compared with the control group and the true-negative group.

Acknowledgements The authors would like to thank the DLCST steering committee, including Jesper Holst Pedersen MD, Asger Dirksen MD, Zaigham Saghir MD, Laura Hohwü Thomsen MD, Martin Døssing MD, Mathilde Marie Winkler Wille MD, Bruno Heleno MD, Thomas Rasmussen MD, Haseem Ashraf MD, Hanne Hansen MD, Birgit Guldhammer Skov MD, Karen Bach MD, Jann Mortensen MD, Hanne Thorsen MD, Niels Seersholm MD, Hanne Hansen MD,
Klaus Fuglsang Kofoed MD, Paul Clementsen MD and Klaus Richter Larsen MD. The authors would like to thank data manager Willy Karlslund (The Research Unit for General Practice and Section of General Practice, Department of Public Health, University of Copenhagen) for the comprehensive technical work generating the databases.

Contributors The study was devised and designed by JB. Data collection was conducted by JB. Statistical analyses were done by JFR and VS. JFR drafted the manuscript and JB, VS and JM contributed to parts of the manuscript and to revisions of the manuscript. All four authors have approved the final version of the manuscript. All authors had full access to all of the data in the study (including statistical reports and tables) and can take responsibility for the integrity of the data and the accuracy of the data analysis.

Funding JFR was funded by the Health Foundation, grant number 2011B179.

Disclaimer The funder had no role in study design or data collection, analysis or interpretation. JB, JM and VS have not received any funding.

Competing interests None declared.

Patient and public involvement Patients and/or the public were involved in the design, conduct, reporting or dissemination plans of this research. Refer to the 'Methods' section for further details.

Patient consent for publication Not required.

Ethics approval The DLCST was approved by the Danish Scientific Ethical Committee (approval number KA-02045). The DLCST was approved by the Danish Data Protection Agency (approval number 2005-53-1083). All participants signed an informed consent form.

Provenance and peer review Not commissioned; externally peer reviewed.

Data availability statement Data are available on reasonable request. The corresponding author can provide the questionnaires and datasets generated and analysed during the study on reasonable request.

Open access This is an open access article distributed in accordance with the Creative Commons Attribution Non Commercial (CC BY-NC 4.0) license, which permits others to distribute, remix, adapt, build upon this work non-commercially, and license their derivative works on different terms, provided the original work is properly cited, appropriate credit is given, any changes made indicated, and the use is non-commercial. See: http://creativecommons.org/licenses/by-nc/4.0/.

ORCID iD

Jessica Malmqvist http://orcid.org/0000-0002-9299-4369 


\section{REFERENCES}

1 Ott JJ, Ullrich A, Mascarenhas M, et al. Global cancer incidence and mortality caused by behavior and infection. J Public Health 2011;33:223-33.

2 Shlomi D, Ben-Avi R, Balmor GR, et al. Screening for lung cancer: time for large-scale screening by chest computed tomography. Eur Respir J 2014;44:217-38.

3 National Lung Screening Trial Research Team, Aberle DR, Adams AM, et al. Reduced lung-cancer mortality with low-dose computed tomographic screening. N Engl J Med 2011;365:395-409.

4 Humphrey LL, Deffebach M, Pappas M, et al. Screening for lung cancer with low-dose computed tomography: a systematic review to update the US preventive services Task force recommendation. Ann Intern Med 2013;159:411-20.

5 Pinsky PF, Church TR, Izmirlian G, et al. The National lung screening trial: results stratified by demographics, smoking history, and lung cancer histology. Cancer 2013;119:3976-83.

6 Manser R, Lethaby A, Irving LB, et al. Screening for lung cancer. Cochrane Database Syst Rev 2013:CD001991.

7 Winkler Wille MM, van Riel SJ, Saghir Z, et al. Predictive accuracy of the PanCan lung cancer risk prediction model -external validation based on CT from the Danish lung cancer screening trial. Eur Radiol 2015;25:3093-9.

8 Field JK, van Klaveren R, Pedersen JH, et al. European randomized lung cancer screening trials: post NLST. J Surg Oncol 2013;108:280-6.

9 Croswell JM, Baker SG, Marcus PM, et al. Cumulative incidence of false-positive test results in lung cancer screening: a randomized trial. Ann Intern Med 2010;152:505-12. W176-80.

10 Barton MB, Moore S, Polk S, et al. Increased patient concern after false-positive mammograms: clinician documentation and subsequent ambulatory visits. J Gen Intern Med 2001;16:150-6.

11 Brodersen J, Jørgensen KJ, Gøtzsche PC. The benefits and harms of screening for cancer with a focus on breast screening. Pol Arch Med Wewn 2010;120:89-94.

12 Brodersen J, Siersma VD. Long-term psychosocial consequences of false-positive screening mammography. Ann Fam Med 2013;11:106-15.

13 Byrne MM, Weissfeld J, Roberts MS. Anxiety, fear of cancer, and perceived risk of cancer following lung cancer screening. Med Decis Making 2008;28:917-25.

14 Goulart BHL, Bensink ME, Mummy DG, et al. Lung cancer screening with low-dose computed tomography: costs, National expenditures, and cost-effectiveness. J Natl Compr Canc Netw 2012;10:267-75.

15 Rasmussen JF, Siersma V, Pedersen JH, et al. Healthcare costs in the Danish randomised controlled lung cancer CT-screening trial: a registry study. Lung Cancer 2014;83:347-55

16 Jensen MD, Siersma V, Rasmussen JF, et al. Direct and indirect healthcare costs of lung cancer CT screening in Denmark: a registry study. BMJ Open 2020;10:e031768.

17 Saghir Z, Dirksen A, Ashraf H, et al. CT screening for lung cancer brings forward early disease. The randomised Danish lung cancer screening trial: status after five annual screening rounds with lowdose CT. Thorax 2012;67:296-301.
18 Brodersen J, Thorsen H, Kreiner S. Consequences of screening in lung cancer: development and dimensionality of a questionnaire. Value Health 2010;13:601-12.

19 van den Bergh KAM, Essink-Bot ML, Borsboom GJJM, et al. Long-term effects of lung cancer computed tomography screening on health-related quality of life: the Nelson trial. Eur Respir $J$ 2011;38:154-61.

20 Gareen IF, Duan F, Greco EM, et al. Impact of lung cancer screening results on participant health-related quality of life and state anxiety in the National lung screening trial. Cancer 2014;120:3401-9.

21 Brain K, Lifford KJ, Carter B, et al. Long-term psychosocial outcomes of low-dose CT screening: results of the UK lung cancer screening randomised controlled trial. Thorax 2016;71:996-1005.

22 McCaffery KJ, Barratt AL. Assessing psychosocial/quality of life outcomes in screening: how do we do it better? J Epidemiol Community Health 2004;58:968-70.

23 Pedersen JH, Ashraf H, Dirksen A, et al. The Danish randomized lung cancer CT screening trial--overall design and results of the prevalence round. J Thorac Oncol 2009;4:608-14.

24 Andersen EB. A goodness of fit test for the Rasch model. Psychometrika 1973;38:123-40.

25 Dufouil C, Brayne C, Clayton D. Analysis of longitudinal studies with death and drop-out: a case study. Stat Med 2004;23:2215-26.

26 Benjamini Y, Hochberg Y. Controlling the false discovery rate: a practical and powerful approach to multiple testing. J R Stat Soc Series B Stat Methodol 1995;57:289-300.

27 Chapple A, Ziebland S, McPherson A. Stigma, shame, and blame experienced by patients with lung cancer: qualitative study. BMJ 2004;328:1470.

28 Hestbech MS, Siersma V, Dirksen A, et al. Participation bias in a randomised trial of screening for lung cancer. Lung Cancer 2011;73:325-31.

29 Grannis FW. Minimizing over-diagnosis in lung cancer screening. J Surg Oncol 2013;108:289-93.

30 Rasmussen JF, Siersma V, Pedersen JH, et al. Psychosocial consequences in the Danish randomised controlled lung cancer screening trial (DLCST). Lung Cancer 2015;87:65-72.

31 Saghir Z, Ashraf H, Dirksen A, et al. Contamination during 4 years of annual CT screening in the Danish lung cancer screening tria (DLCST). Lung Cancer 2011;71:323-7.

32 Lindberg LG, Svendsen M, Dømgaard M, et al. Better safe than sorry: a long-term perspective on experiences with a falsepositive screening mammography in Denmark. Health Risk Soc 2013;15:699-716.

33 Kaerlev L, lachina M, Pedersen JH, et al. CT-Screening for lung cancer does not increase the use of anxiolytic or antidepressant medication. BMC Cancer 2012;12:188.

34 Salz T, Richman AR, Brewer NT. Meta-analyses of the effect of false-positive mammograms on generic and specific psychosocial outcomes. Psychooncology 2010;19:1026-34.

35 Kinsinger LS, Anderson C, Kim J, et al. Implementation of lung cancer screening in the Veterans health administration. JAMA Intern Med 2017;177:399-406. 